\title{
Light on dark
}

\section{Dark energy is a prime target in the proposed US astronomy programme.}

The US National Research Council has published its latest decadal survey, identifying research priorities for astronomy and astrophysics in the years ahead (www.nap.edu/catalog/12951.html). It's an exercise that has taken note of straitened economic circumstances and consulted widely across all of astronomy and astrophysics. Top slot in space has gone to the Wide-Field Infrared Survey Telescope (WFIRST); for large ground-based projects, it's the optical Large Synoptic Survey Telescope (LSST).

It's striking that both WFIRST and LSST are wide-reaching instruments - wide in the area of sky they will survey, wide in the studies they will undertake. Both will target dark energy, the phenomenon invoked to explain the accelerating expansion of the Universe.

These are, of course, only recommendations compiled by representatives of the astronomy community - whether agencies such as NASA decide to run with any of the proposals is another matter. Meanwhile, the European Space Agency (ESA) already has under consideration a proposal for a dark-energy-solving spacecraft, called Euclid; whether it will fly or not is likely to be decided within the next 12 months. NASA and ESA are set to open bilateral talks this month: the quest to understand dark matter would surely be best pursued through international collaboration.

\section{Nicola Cabibbo 1935-2010}

\section{Significant work in particle physics bears his name, but the list of Nobel winners does not.}

Italian particle physicist Nicola Cabibbo died on 16 August 2010. Cabibbo had been a towering figure in Italian physics, particularly as president of the National Institute of Nuclear Physics (INFN) between 1983 and 1992, and then as president of the Pontifical Academy of Sciences. His legacy in particle physics is recorded in the Cabibbo-Kobayashi-Maskawa (CKM) matrix and the Cabibbo angle.

His name did not appear, however, in the list of winners of the 2008 Nobel Prize ${ }^{1}$, which was carried by Makoto Kobayashi and Toshihide Maskawa, alongside Yoichiro Nambu. The prize announcement provoked much comment, and in Italy feelings ran particularly high. Italian newspaper Corriere Della Sera reported Cabibbo's passing under the headline "Death of physicist Cabibbo, who was denied the Nobel".

In the early 1960s, Cabibbo postulated 'weak universality' to make sense of the behaviour of the then-known leptons (two generations) and quarks (only up, down and strange $)^{3}$. For the down and strange quarks, he introduced the Cabibbo angle, which explains the mixing of quarks in terms of a rotation between their mass eigenstates and their weak eigenstates. Kobayashi and Maskawa took these ideas further, adding a third generation of particles to the mix and neatly encapsulating the physics in the $3 \times 3 \mathrm{CKM}$ matrix. Experiments subsequently proved that there are indeed three generations of quark, adding charm, bottom and top to the original cast.
Cabibbo, Kobayashi and Maskawa had looked a natural line-up for the prize, and were much tipped in the field. Certainly it seemed an easier call than the possible future award for the elucidation of the massgenerating mechanism in nature - named for Peter Higgs but to which many parties (and more than the canonical three) can lay claim ${ }^{2}$. There is no doubt whatsoever of Nambu's exceptional contribution to physics, and his Nobel reward was overdue. But the 'sticking together' of Nambu with Kobayashi and Maskawa, thereby forcing the omission of Cabibbo, made the prize seem a little less sweet - and reminiscent of, for example, the 2002 award to Ray Davis, Masatoshi Koshiba and Riccardo Giacconi, all deserving winners but for rather disparate contributions that didn't necessarily sit comfortably together as a single prize, and with the omission of Davis' long-time collaborator John Bahcall.

How much more satisfying those awards that recognize a single, self-contained piece of physics: Albert Fert and Peter Grünberg in 2007 for giant magnetoresistance; or David Gross, David Politzer and Frank Wilczek in 2004 for asymptotic freedom. A one-topic prize is also an easier sell to the media who will publicize the award, no matter how esoteric that one topic might be.

The Nobel committee, of course, have a thankless task. It will always be possible to quibble over who is awarded for what, when and with whom. But the committee members bear well the difficult responsibility of rewarding those who have

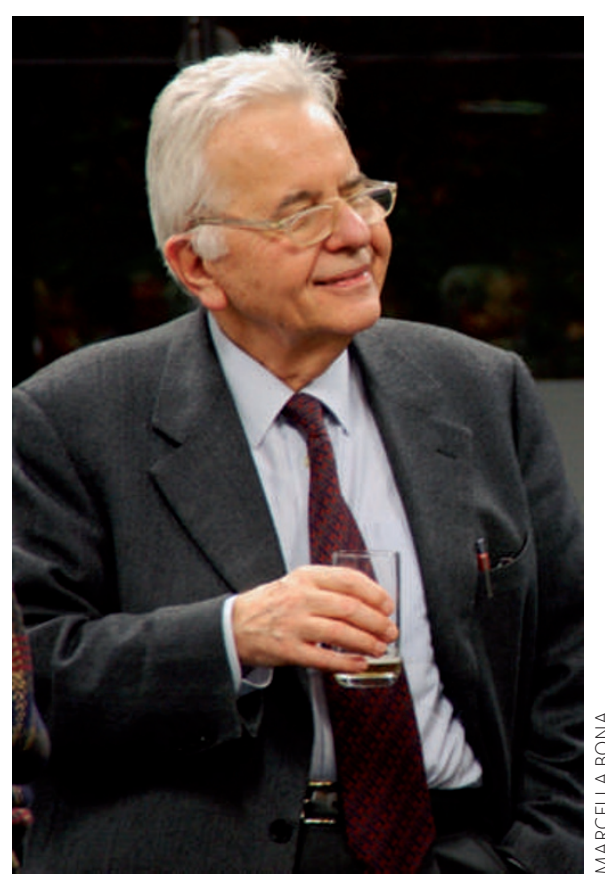

made the truly exceptional contributions to physics, thereby ensuring their legacy in the history of the subject. As ever, we are excited about the next prize announcement, to be made early next month.

Nicola Cabibbo was not a winner, but it would be a shame to let that define his valuable legacy to physics.

\footnotetext{
References

1. http://nobelprize.org/nobel_prizes/physics/laureates/2008/

2. www.nature.com/news/2010/100804/full/news.2010.390.html

3. Cabibbo, N. Phys. Rev. Lett. 10, 531-533 (1963).
} 